\title{
La innovación tecnológica como fuente de competitividad industrial
}

\author{
Technological innovation as a source of industrial \\ competitiveness
}

\begin{abstract}
RESUMEN
El entorno competitivo y cambiante en el cual operan las organizaciones industriales, convierte al proceso de innovación tecnológica como la manera de adaptarse exitosamente a estos cambios, al poder responder de manera oportuna las nuevas necesidades y exigencias del mercado, introduciendo nuevos productos o productos mejorados e implementando nuevos procesos, los cuales significan cambios en los métodos de producción, en la infraestructura, el equipamiento u otros; de manera que todos estos incidan positivamente en la competitividad de las organizaciones industriales. La innovación tecnológica se constituye en un factor primordial, que viene a formar parte del conjunto de activos intangibles que las organizaciones poseen, lo cual les permite anticiparse o responder de manera oportuna a los constantes cambios y exigencias del mercado; este tipo de innovación está constituida por la innovación de productos y por la innovación de los procesos; innovar productos comprende el desarrollo de estos con la consiguiente introducción en el mercado mientras que la innovación de procesos está constituida por el desarrollo y la mejora de los métodos internos de producción que posee una organización. Se aborda a la innovación tecnológica como una forma de diferenciarse de la competencia, mejorar la participación en el mercado, reducir costos, mejorar la calidad, mejorar los procesos existentes, reducir los reprocesos, mejorar la aceptación del producto en el mercado, brindar confianza sobre los productos y los procesos a los clientes, así como a las partes interesadas de la organización.
\end{abstract}

Palabras Clave: Innovación tecnológica; competitividad; organización industrial.

\begin{abstract}
The competitive and changing environment in which industrial organizations operate, makes the technological innovation process the way to successfully adapt to these changes, being able to respond in a timely manner to new market needs and demands, introducing new products or improved products and implementing new processes, which mean changes in production methods, infrastructure, equipment or others; so that all these
\end{abstract}

Javier Francisco Espinoza López jespinoza@iqfarma.com Instituto Quimioterápico S.A. Iqfarma. Lima-Perú

Presentado: 08/10/2020 - Aceptado: 30/04/2021 - Publicado: 23/12/2021

(C) Los autores. Este artículo es publicado por la revista Gestión en el Tercer Milenio de la Facultad de Ciencias Administrativas Universidad Nacional Mayor de San Marcos. Este es un artículo de acceso abierto, distribuido bajo los términos de la licencia Creative Commons Atribución 4.0 Internacional (CC BY 4.0) [https://creativecommons.org/licenses/by/4.0/deed.es] que permite el uso, distribución y reproducción en cualquier medio, siempre que la obra original sea debidamente citada de su fuente original. 
have a positive impact on the competitiveness of industrial organizations. Technological innovation is a primary factor, which becomes part of the set of intangible assets that organizations have, which allows them to anticipate or respond in a timely manner to the constant changes and demands of the market; this type of innovation is made up of product innovation and process innovation; innovating products includes the development of these with the consequent introduction of them into the market, while process innovation is constituted by the development and improvement of the internal production methods that an organization has. Technological innovation is approached as a way to differentiate itself from the competition, improve market share, reduce costs, improve quality, improve existing processes, reduce rework, improve product acceptance in the market, provide confidence about products and processes to customers as well as the organization's stakeholders.

Keywords: Technological innovation; competitiveness; industrial organization.

\section{INTRODUCCIÓN}

El actual entorno en el cual operan las organizaciones industriales en el mundo es cada vez más complejo, dinámico y competitivo; con cambios muy rápidos en la tecnología, en los sistemas de información, en el mercado, en los métodos de trabajo, en las regulaciones, en los modelos de gestión y en las relaciones empresariales; todo ello impulsado por la creciente internacionalización, la integración económica, la apertura de los mercados, la obsolescencia rápida de productos, y el incremento de competidores. Todo esto origina una serie de cambios en la perspectiva económica de las empresas, obligándolas a adaptarse a estas nuevas condiciones, para poder subsistir en el mercado, mejorar su competitividad y hacer frente a la competencia, ya que de otra manera será difícil que puedan sobrevivir (Ríos, 2006).

Asimismo, se percibe una igualdad en la disponibilidad de factores de producción para todos los competidores; lo cual se evidencia por las menores diferencias existentes en los costos de los insumos, en la mano de obra directa, el crédito bancario, el acceso a la tecnología, entre otros; cuyo efecto directo radica en la dificultad y complejidad de competir a través de estas ventajas y por otro lado obliga a las empresas a buscar nuevas formas de mejorar su competitividad, es decir a realizar innovaciones en sus procesos internos o en sus productos de manera que puedan mejorar sus niveles de productividad, rentabilidad y sobre todo diferenciarse de la competencia para obtener una mayor participación en el mercado (Hidalgo et al., 2013).

Por lo tanto, las empresas que serán exitosas son aquellas que puedan adaptarse a los cambios, crear nuevo conocimiento y difundirlos de manera efectiva al interior de toda la organización (Nonaka, 2007), lo cual se pone de manifiesto cuando la empresa tiene la capacidad de gestionar la interacción de las necesidades y oportunidades existentes en el mercado con las oportunidades tecnológicas detectadas, y por lo tanto pueda responder de manera oportuna e inclusive anticiparse a la competencia, al introducir nuevos productos o implantar nuevos métodos de producción (Hinojosa, 2006).

Muchos autores consideran a la innovación tecnológica como una fuente substancial de competitividad para las organizaciones $y$, por otro lado, también constituye un factor importante para el crecimiento y la sostenibilidad de la empresa en el tiempo.

Definitivamente al empresario innovador que busque mejorar la competitividad de su organización le surgirán algunas interrogantes, tales como: ¿Cómo hacer crecer el negocio sin tener que aumentar el tamaño de la empresa? ¿Cómo puede la empresa resistir los cambios inesperados, y las marcadas fluctuaciones del mercado? ¿Cómo reducir costos y, simultáneamente, mejorar la competitividad y diferenciar a la empresa? ¿Cómo reducir costos y 
a la vez incrementar la satisfacción a los clientes? ¿Cómo lograr una mayor participación en el mercado? ¿Cómo mejorar la rentabilidad? ¿Cómo lograr una mayor aceptación de los productos por parte del cliente? (Moraleda, 2004).

Quizás todas estas interrogantes se puedan reducir a una sola y gran pregunta: ¿Cómo hacer que el negocio se diferencie de su competencia, logrando ser el referente en su rubro?

Es evidente que no existe una respuesta académica para cada una de estas preguntas, lo único claro es que las empresas deben abordar un proceso de transformación permanente, que las prepare para afrontar sus retos estratégicos en el contexto de un entorno incierto y cambiante (Moraleda, 2004). Indudablemente, dado que como no existen soluciones preconcebidas, es la innovación tecnológica el camino a través del cual se logrará responder a las interrogantes planteadas y de esta manera obtener las ventajas competitivas que necesita la organización.

\section{OBJETIVO DEL ARTÍCULO}

Resaltar la importancia de la innovación tecnológica como fuente de competitividad y del desarrollo económico de las organizaciones industriales, constituyéndose como un factor que forma parte del conjunto de activos intangibles que las organizaciones poseen, de manera que siempre puedan responder o anticiparse a los cambios permanentes del entorno con el fin de obtener una ventaja competitiva sostenible; está comprendida por la innovación de productos y por la innovación de procesos, la primera consiste en el desarrollo de productos nuevos con la consiguiente introducción de estos en el mercado y la segunda comprende el desarrollo y la mejora de los métodos de producción de la organización.

\section{ARGUMENTACIÓN}

Para entender el significado de innovación tecnológica empezaremos primero entendiendo el significado de la palabra tecnología. Se sabe que en el pasado siempre se ha relacionado a la tecnología con el arte y las máquinas (tecno), relegando los aspectos relacionados a la razón y al conocimiento (logos). Sin embargo, la tecnología va más allá de las máquinas, ya que es conocimiento práctico. Así pues, "tecnología significa aplicación sistemática del conocimiento científico u otro conocimiento organizado a tareas prácticas" (Galbraith, 1980, citado en Fernández y Vásquez, 1996, p. 29).

La tecnología está compuesta en primer lugar por un resultado esperado (la solución a un problema o la mejora de un proceso y/o el desarrollo de un nuevo producto), y en segundo lugar por un conjunto organizado de actividades, es decir, los recursos y la forma para alcanzar ese resultado. Lo cual significa que cualquier tecnología es una relación causa-efecto, que nos permite dar respuesta a la pregunta ¿Qué debemos hacer para lograr el fin perseguido? (Fernández y Vásquez, 1996).

Por otro lado, respecto a la palabra innovación, no existe un acuerdo unánime acerca de su significado, tampoco sobre los tipos de innovación que existen. En lo que sí coinciden los diferentes autores es en relacionar a la innovación con algo nuevo que va de la mano con ideas de cambio (Santos y Álvarez, 2008). Fue el economista austriaco Joseph Schumpeter quien ilustró de modo más certero a la innovación al definirla como la introducción al mercado de un nuevo producto o el cambio cualitativo de uno ya existente, la introducción de un nuevo proceso o método de producción, la apertura de un nuevo mercado, el desarrollo de nuevas fuentes de materia prima, o el cambio en la organización industrial (Schumpeter, 1944).

Es cierto que Schumpeter ve la innovación más desde la perspectiva económica que la productiva, por eso atribuye a la innovación como la responsable del desajuste del equilibrio dinámico del sistema económico. Para este autor, el papel que desempeña la innovación en la evolución y la sostenibilidad de las organizaciones es incuestionable.

El manual de Frascati (1993), citado en Hidalgo et al. (2013) define la innovación tecnológica como la conversión de una idea en un producto, que puede ser nuevo o que haya sido mejorado, y que termina con la introducción de este en el mercado; o en un proceso nuevo o que haya sido mejorado, que es aplicado en las actividades industriales; para este autor, innovar un producto significa la creación de nuevos 
productos, o la mejora de sus características, tales como la calidad o la funcionalidad en relación a los ya existentes, y por otro lado, la innovación de procesos significa la implementación permanente de nuevos procesos de producción o la mejora de los que ya existen, y cuyo objetivo principal es reducir de costos de producción. (Hidalgo et al., 2013).

El manual de Oslo (2005) enmarca a la innovación tecnológica dentro de dos dimensiones, las innovaciones de producto y las innovaciones de proceso. La innovación de producto corresponde a la introducción en el mercado de un bien que puede ser nuevo o que haya sido mejorado de manera substancial; tanto en sus características técnicas, de composición, de documentación, de funcionabilidad o cualquier otra característica propia. Este tipo de innovación puede hacer uso de un conocimiento o una tecnología nueva, o basarse en la utilización o en la combinación de un conocimiento con una tecnología ya existente.

De acuerdo al manual de Oslo (2005) una innovación de proceso a diferencia del producto, corresponde a la introducción de un proceso de producción o de distribución nuevo o mejorado de manera substancial, lo cual conlleva cambios importantes en los métodos de producción, en los materiales, en el equipamiento, en la infraestructura, en los procedimientos de trabajo e inclusive en los programas informáticos. Como ejemplo podríamos mencionar a la introducción de un equipo o sistema automatizado en una línea de producción que busque reducir tiempos de proceso, la instalación de un nuevo software a un equipo para mejorar y controlar el rendimiento de los mismos o la elaboración de nuevos procedimientos de trabajo que busquen dinamizar los procesos de producción a fin de hacerlos más eficientes. Otro ejemplo podría ser la modificación necesaria al método de producción para adaptarlo al uso de un nuevo insumo; o cualquier otro cambio para cumplir con un requisito regulatorio, normativo o exigido por el cliente.

El objetivo de la innovación de un proceso puede ser la disminución de costos de producción, mejorar la calidad del producto, mejorar los procesos existentes, reducir los reprocesos y retrabajos, mejorar la aceptación del produc- to por parte del cliente, brindar confianza sobre los productos que hacen uso de estos nuevos procesos a los clientes internos y externos, así como a las partes interesadas de la organización (Manual de Oslo, 2005).

Respecto a la innovación tecnológica, esta podría definirse como "el hecho de comercializar por primera vez una tecnología en el mercado. Esta puede ser tanto de producto como de proceso" (Fernández y Vásquez, 1996, p. 30), y es este un proceso estratégico que con mayor frecuencia se aplica en las organizaciones industriales ya que para el negocio constituye una fuente de competitividad.

La innovación tecnológica por lo tanto viene a ser el conjunto de actividades (científicas, técnicas, comerciales, organizacionales, financieras y otras) a través del cual una empresa implanta mejoras sustanciales en el proceso productivo y en sus productos, creando de esta manera nuevas dimensiones de desempeño de los mismos. Todo ello en base a la gestión de la interacción entre las necesidades existentes del mercado, las oportunidades tecnológicas detectadas y la capacidad que tiene la empresa, para responder o incluso anticiparse a estas necesidades y oportunidades en base a la introducción de nuevos productos al mercado o a la implantación de nuevos métodos de producción (Hinojosa, 2006).

Es importante resaltar que si un proceso de innovación tecnológica, no culmina con la colocación del producto servicio en el mercado, o en la utilización efectiva del nuevo proceso desarrollado en las operaciones de la empresa, estaremos frente a una actividad creativa, de invención o de investigación, mas no de una innovación tecnológica.

Algo adicional que menciona Hinojosa (2006) es que un proceso de innovación tecnológica debe crear nuevas dimensiones de desempeño, esto significa que se necesita que exista una forma de medir el comportamiento del nuevo producto en el mercado y de qué manera es aceptado por el cliente y para el caso del nuevo proceso de producción, el desempeño sería la medida de cómo es aceptado o percibido este proceso por parte de los empleados que hacen uso de los mismos. 
Una definición concreta del significado de la innovación tecnológica desde la perspectiva administrativa vendría a ser la siguiente: lograr la planificación, organización, control y dirección de los recursos internos de una organización, tanto económicos, así como los ligados al conocimiento, con el fin de generar y promover la creatividad tecnológica que permitan desarrollar nuevos productos y nuevos procesos o mejorar los existentes, de manera de obtener un resultado comercial que genere valor a la organización industrial (Roberts, 1987, citado en Petrillo et al., 2013).

El desarrollo de nuevos productos es una oportunidad de innovación ofensiva ya que parte de la identificación de una necesidad del cliente y del mercado; y la innovación de procesos es una oportunidad de innovación defensiva ya que parte por identificar oportunidades de mejoras en los métodos de producción al interior de las organizaciones industriales (Bermúdez, 2013). Ambos tipos de innovación son la fuente de la competitividad industrial.

Al resaltar que la innovación tecnológica constituye la fuente para la competitividad industrial, hará que esta dependa de aspectos tales como la calidad y la novedad de los productos ofertados al mercado, los métodos de producción utilizados, la manera como se organiza la producción, los tiempos de entrega del producto al cliente, el nivel de participación en el mercado e inclusive el nivel de atención y la calidad del servicio postventa (Molina y Conca, 2000).

Comprender la manera de cómo gestionar la innovación tecnológica con éxito es de vital importancia ya que esta se constituye en una estrategia de supervivencia casi obligatoria para las organizaciones industriales (Ballinas y Solleiro, 2013). Recordemos la célebre frase "innovar o morir" (Drucker, 1999 citado en Ballinas y Solleiro 2013, p. 5), cuyo significado nos lleva a concluir que una organización que no innova sus productos y sus procesos de manera continua, está condenada al fracaso y por ende de manera inevitable a la desaparición.

De todo lo expuesto podemos deducir que la gestión de la innovación tecnológica hace uso de herramientas administrativas, las mismas que las complementa con el uso del conocimiento para llevar a cabo procesos de transformación que permitan obtener nuevos productos y nuevas formas de producción o, dicho de otra manera, nuevos procesos productivos en todas sus fases; las mismas que surtirá efecto si se gestionan de manera permanente o continua para que puedan agregar valor a las organizaciones industriales y hacerlas más competitivas.

Respecto a la competitividad, Porter y Millar (1985) señalan que "la competitividad es la capacidad de una empresa para producir y mercadear productos en mejores condiciones de precio, calidad y oportunidad que sus rivales" (p. 3). Por otro lado, "las empresas crean ventaja competitiva al percibir o descubrir nuevas y mejores formas de competir en un sector y trasladarlas al mercado, lo que en último extremo es un acto de innovación" (Porter, 1998, p. 83).

La competitividad entonces es un concepto dinámico que va evolucionando en el tiempo, pues este va a depender del estado cambiante del entorno y del mercado; ya que los factores que en algún momento contribuían al crecimiento empresarial, dejan de ser importantes cuando surgen nuevas exigencias y nuevas necesidades, exigiendo a las organizaciones cambios y adaptaciones en sus prácticas de gestión. La competitividad entonces como concepto permite a las organizaciones industriales evaluar su nivel de crecimiento y desarrollo empresarial (Estrada et al., 2009).

Nótese que, de acuerdo a los autores citados, podemos concluir que la innovación tecnológica se enmarca dentro de dos dimensiones, la innovación de productos (lanzamiento al mercado de productos nuevos o con nuevas características de desempeño para su comercialización) y la innovación permanente de los procesos de producción (adopción o implantación de métodos o técnicas de producción que pueden ser nuevos o pueden tener cambios en su desempeño que le permita a la empresa mejorar sus operaciones y hacerla más eficiente).

Para que lo anterior ocurra será necesario que las empresas en primer lugar deban identificar, desglosar y definir sus principales procesos empresariales; para luego articular los elementos claves de la innovación en cada proceso 
particular que se considere importante de innovar (Mathison et al., 2007). Todos los procesos tienen un valor real cuando están relacionados en integrados unos con otros, conformando la cadena de valor de las organizaciones.

Adicionalmente es importante sumar a las dos dimensiones antes mencionadas dos procesos adicionales, la estrategia tecnológica y la adquisición de tecnología (Mathison et al., 2007), de manera de contar con cuatro procesos industriales típicos, los cuales deben funcionar de manera paralela para lograr una óptima competitividad.

Mathison et al. (2007) considera estos cuatro procesos de innovación tecnológica como dos parejas que actúan de manera simbiótica.

- Las primeras dos están conectadas considerando a la innovación tecnológica como un resultado: Desarrollo de nuevos productos y la innovación de procesos. Ambas se circunscriben en la aplicación o explotación de la tecnología.

- Los otros dos son necesarios para que las anteriores tengan éxito: la estrategia tecnológica y la adquisición de tecnología (esta última puede ser generada al interior de la organización o adquirida de algún proveedor). Este grupo se sustenta en la capacidad de la organización para planificar, controlar y ejecutar la tecnología, tal como podemos observar en la Figura 1.

Estos cuatro procesos deben estar integrados a la estructura general de la organización ya que no pueden gestionarse de manera independiente. Es decir, deben tener soporte a través de la gestión de los recursos humanos, los sistemas implementados para el control de los recursos financieros, los asuntos legales y reglamentarios, el sistema de gestión de calidad, el sistema de gestión medioambiental, el sistema de seguridad y salud ocupacional o cualquier otro relacionado; todos ellos encaminados con una visión de liderazgo (Mathison et al., 2007).

Es cierto que los procesos antes mencionados dependerán del tamaño, tipo, cultura organizacional, visión empresarial y el giro de negocio; pero a medida que una empresa se diferencie de la competencia, mejore su participación en el mercado, incremente su tamaño y por ende su patrimonio; las prácticas administrativas de sus procesos internos requerirán de

\section{Figura 1}

Procesos para la innovación tecnológica

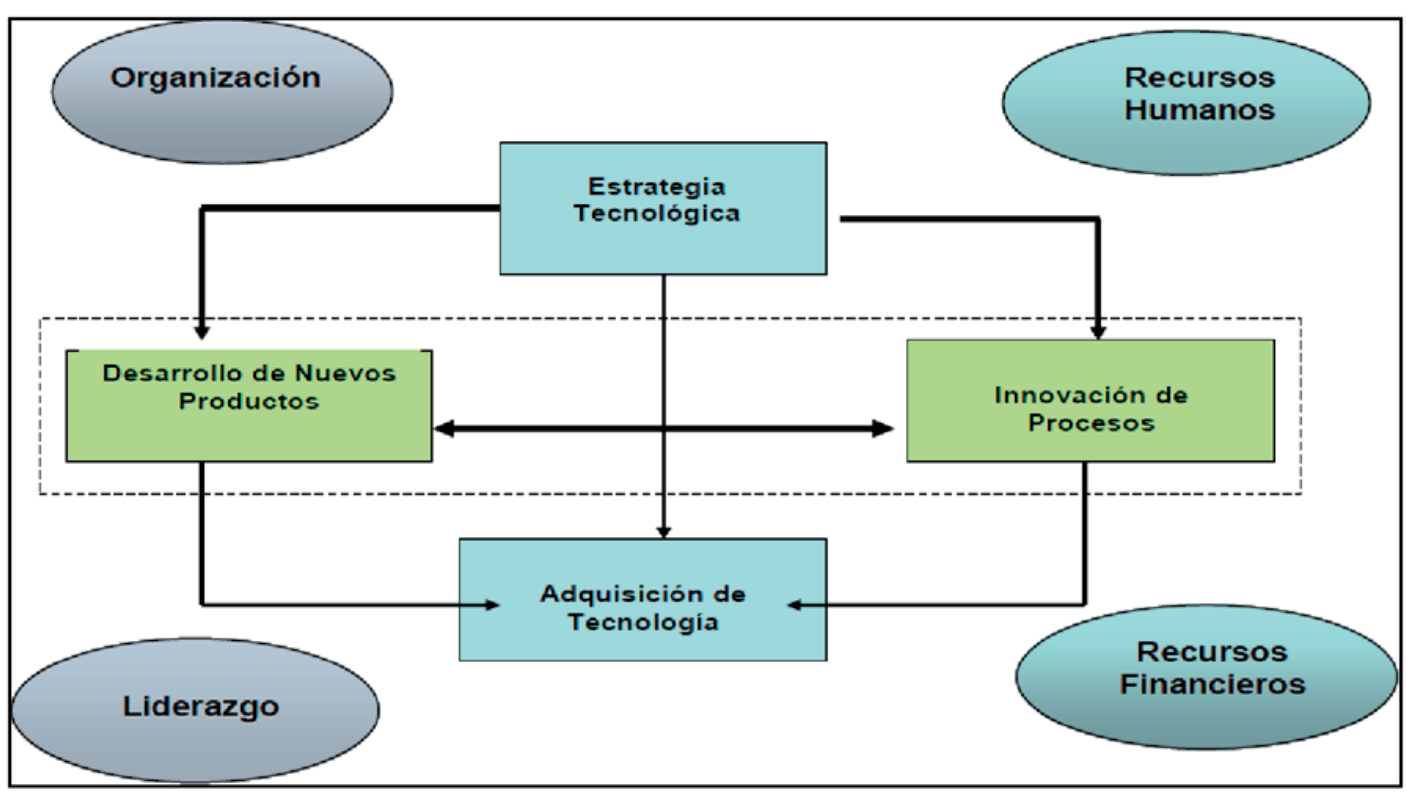

Nota. Adaptado del libro “Innovación Factor Clave para lograr Ventajas Competitivas”, por Mathison et al., 2007, p.78. 
una mayor atención, haciendo que la innovación tecnológica deje de ser tratada como un proceso adicional a los existentes y se convierta en un proceso estratégico que brinde soporte y sea la fuente de la competitividad empresarial.

En el Perú los resultados obtenidos de la Encuesta Nacional de Innovación en la Industria Manufacturera del año 2015, muestran que las empresas industriales que tienen éxito gestionando actividades de innovación tecnológica según actividad económica pertenecen mayormente al rubro de fabricación de otro equipo de transporte con $77,5 \%$ de empresas innovadoras, seguido por el rubro de elaboración de productos alimenticios con 73,5\%; el de elaboración de bebidas con 67,6\%; y finalmente los del rubro de fabricación de productos farmacéuticos y medicinales con $66,0 \%$. Algo que es importante resaltar es que el $72.3 \%$ de las actividades de innovación tecnológica que realizaron todas ellas, estuvo orientada a la adquisición de bienes de capital para llevar a cabo los procesos de producción y formar parte de sus patrimonios (Instituto Nacional de Estadística e Informática, 2017).

Como ejemplos de empresas innovadoras exitosas tanto a nivel nacional como internacional según actividad económica tenemos al SIMA conocido también como Servicios Industriales de la Marina (correspondiente al sector de fabricación de otro equipo de transporte); las empresas ALICORP y GRUPO GLORIA (del sector de elaboración de productos alimenticios) las empresas AJE GROUP y UCP BACKUS \& JOHNSTON (sector de elaboración de bebidas) y las empresas IQFARMA Y MEDIFARMA (del sector de fabricación de productos farmacéuticos y medicinales).

\section{CONCLUSIONES}

La innovación tecnológica como proceso estratégico de las organizaciones industriales incide en la competitividad de estas, al conseguir la introducción de nuevos productos en el mercado, y las mejoras sustanciales en sus procesos productivos. Este proceso inicia con la identificación de las necesidades existentes en el mercado, y de las oportunidades tecnológicas detectadas en el entorno o al interior de la organización, las cuales interactúan con la capacidad que posee la empresa para responder o anticiparse a las necesidades identificadas; ya sea introduciendo nuevos productos al mercado que logren la aceptación de los clientes o bien implementando nuevos métodos de producción o mejorando sustancialmente los ya existentes, y logrando que estos sean aceptados por los miembros de la organización que hacen uso de los mismos.

La innovación tecnológica, hace uso de herramientas administrativas, las que complementa con el uso del conocimiento, que permiten por un lado innovar los productos, cuyo objetivo puede ser el de mejorar sus características técnicas, de composición, de funcionalidad, diferenciarse de la competencia, permanecer en el mercado o mejorar su participación en el mercado y por otro lado tenemos a la innovación de procesos cuyo objetivo puede ser la disminución de costos de producción, mejorar la calidad del producto, dinamizar las líneas de producción, reducir los reprocesos, mejorar los procesos existentes, mejorar la aceptación del producto por parte del cliente y algo muy importante que consiste en brindar confianza sobre los productos que hacen uso de estos nuevos procesos a los clientes internos y externos, así como a las partes interesadas de la organización.

Las actividades de innovación tecnológica constituyen un elemento de entrada para realizar investigaciones mucho más profundas en las empresas manufactureras nacionales, acorde a las actividades económicas o sector industrial a las cuales pertenecen; esto permitirá conocer mucho más de cerca la incidencia de todas estas actividades en la competitividad de cada una de ellas.

\section{REFERENCIAS BIBLIOGRÁFICAS}

Ballinas, M., y Solleiro, J. (2013). La Gestión de la Innovación en empresas farmacéuticas mexicanas. Ciudad de México, México: ANFECA.

Bermúdez, J. (2013). Como medir la innovación en las organizaciones. Lima, Perú: Cuadernos de Investigación UPC.

Estrada, R., García, D., y Sánchez, V. G. (2009). Factores determinantes del éxito en la Pyme. Estudio Empírico en México. Revista Venezolana de Gerencia, 14 (46), 169-182.

Fernández, E., y Vásquez, C. J. (1996). El Proceso de Innovación Tecnológica en la Empresa. Investigaciones Europeas de Dirección y Economía de la Empresa, 2 (1), 29-45. 
Hidalgo, A., León, G., y Pavón, J. (2013). La gestión de la innovación y la tecnología en las organizaciones. Madrid, España: Pirámide.

Instituto Nacional de Estadística e Informática (2017). Encuesta Nacional de Innovación en la Industria Manufacturera 2015. Lima: Instituto Nacional de Estadística e Informática.

Hinojosa, A. (2006). Innovación de proceso. Ciudad de México, México: Cuadernos de gestión de tecnología.

Manual de Oslo. (2005). Guía para la recogida e interpretación de datos sobre innovación. Países miembros de la OCDE: OECD/European communities.

Mathison, L., Gándara, J., Primera, C., y García, L. (2007). Innovación: Factor Clave para lograr Ventajas Competitivas. NEGOTIUM, 3 (7), 46-83.

Molina, H., y Conca, F. (2000). Innovación Tecnológica y Competitividad Empresarial. Alicante, España: Publicaciones de la Universidad de Alicante.

Moraleda, A. (2004). La innovación, clave para la competitividad empresarial. Universia Business Review, 1(6), 128-136.

Nonaka, I. (2007). La empresa creadora de conocimiento. Harvard Business Review América Latina, 1-9. Recuperado de https://bschogardecristo. files.wordpress.com/2007/08/nonaka_red.pdf
Petrillo, J. D., Doumecq, J. C., Petrillo, M. I., y Morcela, A. O. (2013). La Gestión de la Innovación Tecnológica y el desarrollo territorial. Red Emprendesur, 1-27. Recuperado de https://www. researchgate.net/publication/259282203_La_ Gestion_de_la_Innovacion_Tecnologica_y_el_ Desarrollo_Territorial_GIT

Porter, M. E. (1998). The Competitive Advantage of Nations. New York, USA: The free press.

Porter, M. E., y Millar, V. E. (1985). How Information Gives You Competitive Advantage. Harvard Business Review, 1-13. Recuperado de https://hbr.org/1985/07/how-information-gives-you-competitive-advantage

Ríos, J. (2006). El entorno empresarial y la estrategia. Management \& empresa, 1-8. Recuperado de http://biblioteca.esucomex.cl/RCA/El\%20 entorno\%20empresarial\%20y\%20la\%20estrategia.pdf

Santos, M., y Álvarez, L. (2008). Efectos de la Gestión de Calidad Total en la transformación en la Innovación Tecnológica y Administrativa. Cuadernos de Economía y Dirección de la Empresa, 1(37), 33-66.

Schumpeter, J. A. (1944). Teoría del Desenvolvimiento Económico. Madrid, España: Fondo de cultura económica 